\title{
Endoscopic submucosal dissection of tracheal deep benign fibrous histiocytoma using hybrid knife
}

This article was published in the following Dove Press journal: OncoTargets and Therapy

Jiang-wei Ma'
Yuan Miao ${ }^{2}$
Xu-yong Lin'
Qiu-yue Wang'
Wei Wang'
Jian Kang'
Gang Hou'
'Department of Pulmonary and Critical
Care Medicine, First Hospital of China
Medical University, Shenyang, People's
Republic of China; ${ }^{2}$ Department of
Pathology, The First Hospital and College
of Basic Medical Sciences, China Medical
University, Shenyang, People's Republic of
China

Correspondence: Gang Hou Department of Pulmonary and Critical Care Medicine, First Hospital of China Medical University, I I000 I Shenyang, People's Republic of China

Tel +86 2483282530

Fax +86 2483282002

Email hougangcmu@163.com

\begin{abstract}
Deep benign fibrous histiocytoma $(\mathrm{FH})$ is an uncommon and poorly recognized tumor that arises in subcutaneous or deep soft tissue. Deep benign FH of the trachea is even more rare. Deep benign FH recurs in approximately $20 \%$ of cases, so surgical resection is the recommended treatment for FH. However, some patients are afraid of the trauma and potential complications from the resection of tracheal tumors, so they do not accept surgical resection. With the development of interventional pulmonology, bronchoscopic intervention plays a key role in the treatment of benign tracheal tumors. Novel tools have emerged in interventional pulmonology and optimized the traditional techniques. Here, we report a case of deep benign $\mathrm{FH}$ of the trachea with broad base relapse after electrocautery with snare, argon plasma coagulation (APC) and radiotherapy. It was treated successfully by endobronchial resection with a hybrid knife (HK) and APC. The HK is a hybrid device that combines an electrocautery knife with a water jet in one instrument. Submucosal injection and circumferential cutting and dissection of lesions as well as coagulation of bleeding can be performed only with the HK. During the 6 months follow-up, the deep benign FH of the trachea did not relapse. In the case reported herein, the HK enabled submucosal injection and circumferential cutting and dissection of lesions as well as access to submucosal lesions to gain further adequate APC treatment, thereby allowing a better clinical response than that achieved by traditional electrocautery with snare and APC. In conclusion, the HK with a water jet is a feasible and effective endoscopic method for management for benign or lowgrade malignant neoplasms of the trachea, especially those with a wide base and submucosal involvement.
\end{abstract}

Keywords: tracheal tumors, interventional pulmonology, fibrous histiocytoma, electrocautery, hybrid knife

\section{Introduction}

Benign fibrous histiocytoma $(\mathrm{FH})$ is one of the most common mesenchymal neoplasms of the skin. Deep benign $\mathrm{FH}$ is an uncommon and poorly recognized clinical subtype that arises in subcutaneous or deep soft tissue. ${ }^{1}$ Tracheal deep benign $\mathrm{FH}$ is rare. Limited information is available concerning the clinical behavior of deep FH due to the paucity of the literature. Although the preferred therapy is surgery for such benign tracheal tumors, bronchoscopic interventions are important and effective alternatives. ${ }^{2-4}$

A variety of techniques, such as electrocautery, argon plasma coagulation (APC), laser, and cryotherapy through a flexible or rigid bronchoscope, have been 
applied to treat tracheal-bronchial tumors. However, electrocautery is suitable for tumors with peduncular structures and limited in tumors with a wide base. For tracheal tumors with a wide base and membranous wall involvement, the depth of APC treatment is approximately 3-4 mm (although relatively safer but not deep enough for elimination of the tumor), and treatment with laser has the risk of perforation. The hybrid knife (HK) treatment was first applied in the endoscopic submucosal dissection (ESD) of gastrointestinal neoplasms. It is a hybrid device that combines an electrocautery knife with a water jet in one instrument. It should simplify and accelerate ESD, because submucosal injection and circumferential cutting and dissection of lesions as well as coagulation of bleeding can be performed with the same device. ${ }^{5}$ The membrane wall is a special portion of the trachea with a continuous muscular layer, which enables the application of the HK for submucosal injection, circumferential cutting and dissection of lesions, and exposure of submucosal lesions to gain further adequate treatment.

Here, we reported a case of deep benign FH of the trachea with a broad base relapse after electrocautery with snare, APC and radiotherapy. It was treated successfully by HK and APC without relapse. The patient provided written informed consent to have the case report and any accompanying images published. Institutional approval was obtained from the Institutional Ethical Review Board of First Hospital of China Medical University to publish the case details.

\section{Case report}

A 32-year-old female was admitted to our department for recurrent tracheal tumor. Half a year ago, she presented with progressive exertional dyspnea and wheezing for 1 month. A chest computed tomography (CT) revealed an endotracheal mass of $11 \mathrm{~mm}$ in diameter and approximately $30 \mathrm{~mm}$ from the carina (Figure 1A). The patient underwent bronchoscopy that showed a well circumscribed posterior soft mass occluding over $90 \%$ of the tracheal lumen (Figure 1B). The patient refused surgical resection of the trachea and accepted bronchoscopic treatment with electrocautery snare and APC (Figure1C).

Histopathological findings: sections from routine formalin fixation and paraffin embedded tissue stained with hematoxylin and eosin revealed ovoid to spindle shaped histiocytes in a storiform pattern accompanied by stromal fibrous tissue hyperplasia with sclerosis (Figure1D, E), Immunohistochemically tumor cells were positive for vimentin and CD68 (Figure 1F, G), negative for CD34 and epidermal membrane antigen (EMA). Hence, the histology was in consistent with the diagnosis of deep benign fibrous histiocytoma. She also accepted radiotherapy after endobronchial resection with $60 \mathrm{~Gy} / 30 \mathrm{f}$. Unfortunately, 6 months later, tumor recurrence was observed (Figure 2A, B). Considering the insufficient depth of the electrocautery snare and APC treatment may have contributed to the relapse, with full informed consent, the HK was used to dissect the tumor and expose the lesion located in the basal layer. Tumor basal depth and blood flow was assessed by endobronchial ultrasound (EBUS) before treatment (Figure 2C). We used a water jet HK (Erbe Hybrid Knife ${ }^{\circledR}$ T-type I-jet, ERBE Elektromedizin) to perform electrosurgical circumferential cutting and dissection of the base of the tracheal lesion, and we used a water jet to inject a solution of $2 \%$ lidocaine into the submucosal layer with 30 Bar pressure (Figure 2D, E). After making a pinhole in the margin of the elevated mucosa with the HK (Figure 2F), the tip of the HK was inserted into the hole, and a circumferential incision was made (VIO mode ENDO CUT Q, effect3-width1-interval of incision4, Coagulation effect 2 and 30W) (Figure 2G, H). Finally, the incised piece of lesion was collected using a cryoprobe. The lesion in the submucosal layer was fully exposed and then treated with APC (Figure 2I, J). The pathological result was still benign FH. There was no recurrence at 9 months followup on bronchoscopy and chest CT (Figure $2 \mathrm{~K}-\mathrm{M}$ ).

\section{Discussion}

FH is a mesenchymal tumor first described by O'Brien and Stout $^{6}$ and occurs predominantly in the soft tissue and bones. Tracheal FH is very rare. Due to the limited number of cases in the literature, the biological behavior of this tracheal neoplasm is unclear at present. A complete analysis suggested that this neoplasm might be evaluated as a low-grade malignancy. ${ }^{7}$

Surgical resection is the recommended treatment for $\mathrm{FH}$ because local recurrence may occur if the tumor is incompletely excised. ${ }^{8-10}$ Deep benign $\mathrm{FH}$ recurs in approximately $20 \%$ of cases, and metastases are rare. ${ }^{1}$ Some patients are afraid of the trauma and potential complications of the surgical resection of tracheal tumors, so they do not accept surgical resection, and some patients with long lesion cannot accept surgical resection. With the development of interventional pulmonology, bronchoscopic intervention plays a key role in treating benign tracheal tumors. ${ }^{11-13}$ Conforti et $\mathrm{al}^{14}$ reported a case of tracheal FH in an infant successfully treated by endoscopy and Nd:YAG(neodymium:yttrium alluminum 


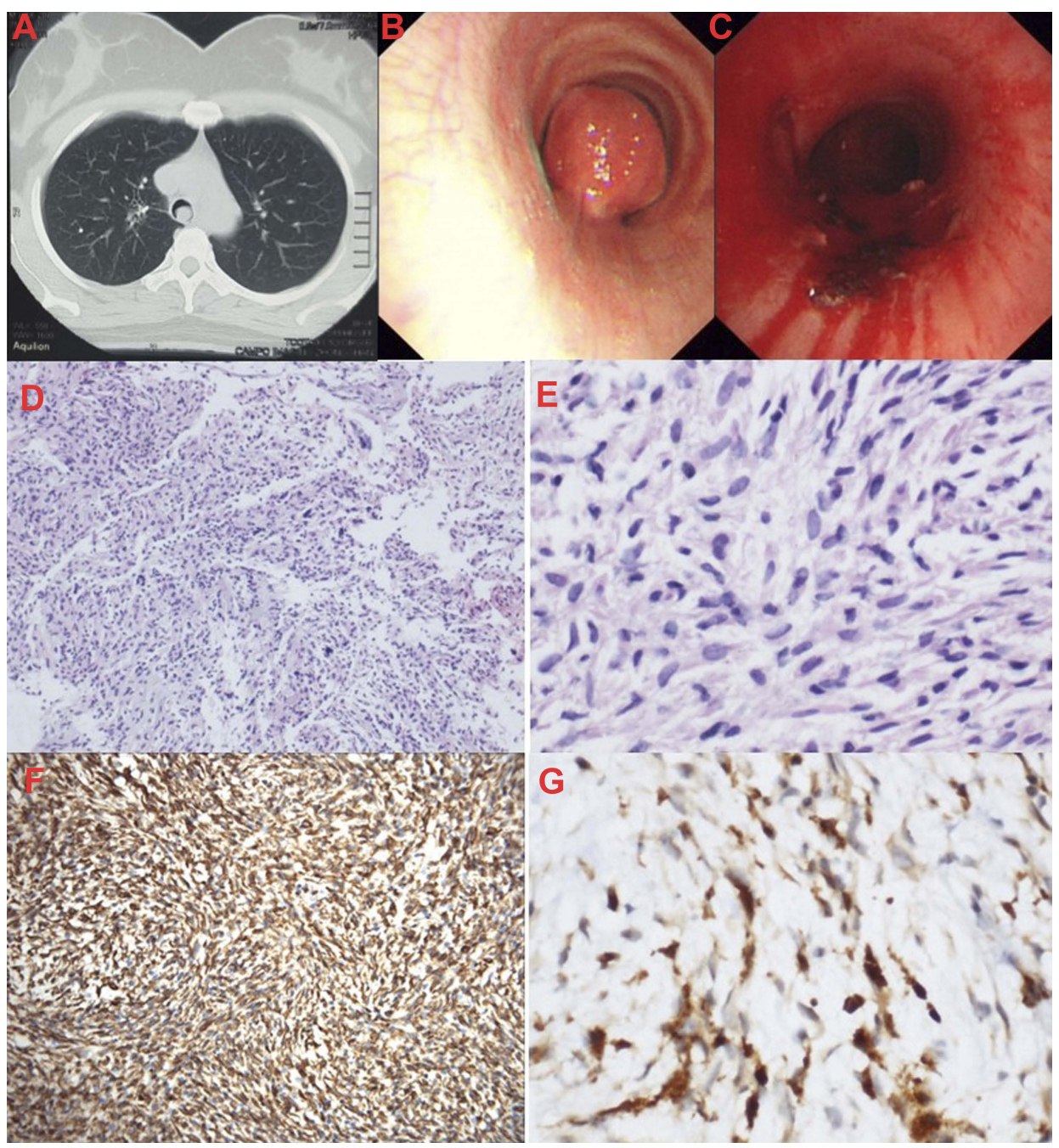

Figure I (A) Chest CT revealed an endotracheal mass of II mm in diameter. (B) Bronchoscopy showed a well circumscribed posterior soft mass occluding over $95 \%$ of the tracheal lumen. (C) The endotracheal mass was treated with electrocautery snare and APC via bronchoscope. (D) Histopathology of the tumor: spindle shaped histiocytes in a storiform pattern accompanied by stromal fibrous tissue hyperplasia with sclerosis, H\&E I00 × . (E) H\&E $400 \times$. (F) Immunohistochemical staining, Vimentin (+). (G) CD68 (+). Abbreviations: CT, computed tomography; APC, argon plasma coagulation; H\&E, hematoxylin and eosin.

garnet) laser. Because a few of the benign or low-grade malignant tumors are prone to recur or change to malignancy, it is very important to repeat bronchoscopies for follow-up. We must carefully practice interventional bronchoscopy to avoid hampering possible surgery in the future. ${ }^{15}$ Our patient accepted electrocautery and APC, but the tumor relapsed shortly after. We considered the main cause of the local recurrence was due to the inadequate depth and width of the endoscopic treatment, and the elimination of the lesions located in the submucosal layer was insufficient. Worrying about the risk of tracheal perforation with the laser, we used the HK as a treatment option. The HK is a novel electrosurgical instrument, which combines submucosal injection and electrocautery. The feasibility of this new device has been assessed in animal stomach, esophagus and colon and there are quite a number of initial experiences in humans. ${ }^{16,17}$ Besides the treatment of early stage gastrointestinal tumors, the hybrid knife has been reported in en bloc transurethral resection of bladder tumour which is nonmuscle invasive, ${ }^{18}$ and treatment of primary tracheal mucosa-associated lymphoid tissue lymphoma, ${ }^{19}$ also for the diagnosis of pleural effusions. ${ }^{20}$ Based on the previous experience of using a HK via semirigid thoracoscopy for electrocautery biopsy of the pleura, ${ }^{20}$ we hypothesized that the HK could make the dissection of a tracheal lesion large and deep enough without obvious damage because of the submucosal water cushion, making APC treatment of the fully exposed base more effective. The clinical response of our case supported our hypothesis. Our case demonstrated that the HK is a potential tool for sufficient resection of benign or low-grade malignant tracheal 


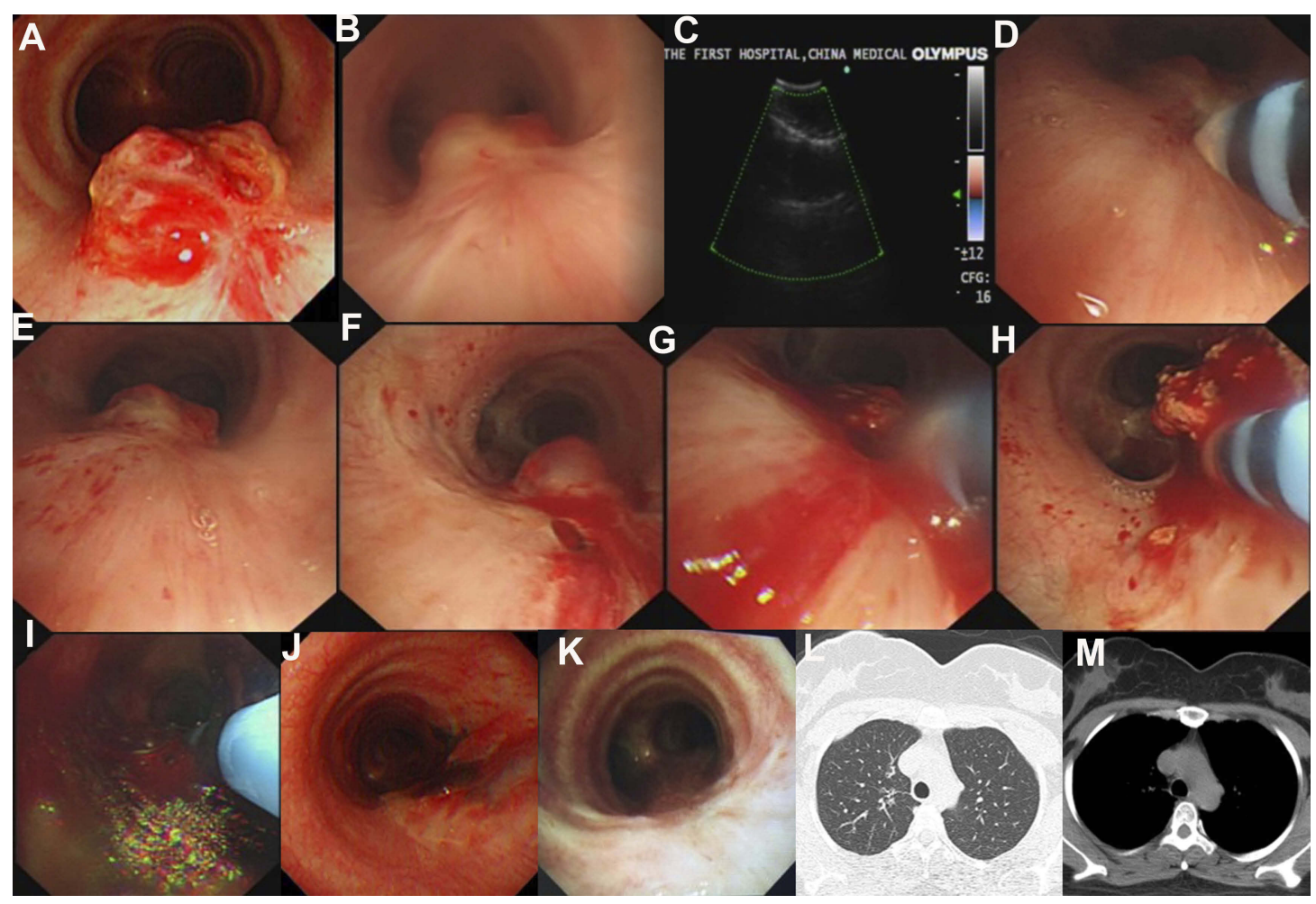

Figure 2 (A) Six months after first bronchoscopic treatment, tumor recurrence at the original site was observed. (B-I) Procedure of treatment with HK. (B) Bronchoscopic view before treatment. (C) EBUS assessment before treatment. (D) The high-pressure water jet (30 Bar) positioned near the tumor and injected a solution of $2 \%$ lidocaine into the submucosal layer. (E) The base of the tumor was elevated. (F) A pinhole of the mucosa with the HK. (G, H) HK was inserted into the hole, and a circumferential incision was made. (I) APC was performed to facilitate the ablation of the tumor base. (J) Bronchoscopic view at the third day after bronchoscopic treatment. (K) Nine months follow-up, bronchoscopy revealed no recurrence. (L,M) Chest CT on ninth month follow-up.

Abbreviations: HK, hybrid knife; EBUS, endobronchial ultrasound; APC, argon plasma coagulation; CT,computed tomography.

tumors with a wide base and membranous wall involvement, the base can be completely elevated and dissected and exposure of submucosal lesions to gain further adequate APC treatment.

The most common complications in the treatment of tumors of the membranous trachea are bleeding and perforation. The combination of electrocautery and electrocoagulation may decrease the risk of bleeding. The HK can create an adequate submucosal cushion, and the direction of dissection can be targeted tangentially to the surface of the lesion at the submucosal layer to minimize the risk of perforation. ${ }^{5}$ Additionally, the thermal and mechanical damage of the resection bed is less pronounced because of the tissue-sparing blunt dissection of the submucosa by the higher amount of injected fluid and the water jet $\mathrm{HK}^{5}$

However, there are several limitations. We reported only one case, and more cases of benign or low-grade tracheal malignancies should be evaluated. In addition, long-term follow-up should be performed to observe the long-term efficacy of the HK method.

\section{Conclusion}

The HK is a novel tool for endoscopic management of benign or low-grade malignant tracheal neoplasms, especially those with a wide base and submucosal involvement.

\section{Disclosure}

The authors report no conflicts of interest in this work.

\section{References}

1. Gleason BC, Fletcher CD. Deep "benign" fibrous histiocytoma: clinicopathologic analysis of 69 cases of a rare tumor indicating occasional metastatic potential. Am J Surg Pathol. 2008;32(3):354-362. doi:10.1097/PAS.0b013e31813c6b85

2. Guo L, Wang K, Zhu H, Liu N, Zhu D. Treatment of primary tracheal glomus tumors: two case reports and a literature review. Medicine (Baltimore). 2018;97(15):e0374. doi:10.1097/MD.0000000000010374

3. Scarlata S, Fuso L, Lucantoni G, et al. The technique of endoscopic airway tumor treatment. $J$ Thorac Dis. 2017;9(8):2619-2639. doi: $10.21037 /$ jtd.2017.07.68

4. Rabeau A, Mazieres J, Hermant C, Projetti F, Didier A, Guibert N. Bronchoscopic multimodal management of tracheal neurofibroma. J Bronchology Interv Pulmonol. 2016;23(4):340-342. doi:10.1097/ LBR.0000000000000220 
5. De-la-Pena J, Calderon A, Esteban JM, et al. Experimental study of hybrid-knife endoscopic submucosal dissection (ESD) versus standard ESD in a Western country. Rev Esp Enferm Dig. 2014;106(2):98-102.

6. O'Brien JE, Stout AP. Malignant fibrous xanthomas. Cancer. 1964;17:1445-1455. doi:10.1002/1097-0142(196411)17:11<1445:: aid-cncr2820171112>3.0.co;2-g

7. Ozturk S, Kutlay H, Sak SD, Yavuzer S. Fibrohistiocytic tumor of the trachea in a child. Eur J Cardiothorac Surg. 1999;16(4):464-468. doi:10.1016/s1010-7940(99)00288-2

8. Sandstrom RE, Proppe KH, Trelstad RL. Fibrous histiocytoma of the trachea. Am J Clin Pathol. 1978;70(3):429-433. doi:10.1093/ajcp/ 70.3.429

9. Gonzalez-Campora R, Matilla A, Sanchez-Carrillo JJ, Navarro A, Galera H. 'Benign' fibrous histiocytoma of the trachea. J Laryngol Otol. 1981;95(12):1287-1292.

10. Sculerati N, Mittal KR, Greco MA, Ambrosino MM. Fibrous histiocytoma of the trachea: management of a rare cause of upper airway obstruction. Int J Pediatr Otorhinolaryngol. 1990;19(3):295-301.

11. Handa A, Fujita K, Yamamoto Y, Komori K, Miyakawa T. Tracheal Tumor. J Pediatr. 2016;173:262-262 e261. doi:10.1016/ j.jpeds.2016.03.017

12. Isaac BT, Christopher DJ, Thangakunam B, Gupta M. Tracheal schwannoma: completely resected with therapeutic bronchoscopic techniques. Lung India. 2015;32(3):271-273. doi:10.4103/0970-2113.156252

13. Miller SM, Bellinger CR, Chatterjee A. Argon plasma coagulation and electrosurgery for benign endobronchial tumors. J Bronchology Interv Pulmonol. 2013;20(1):38-40. doi:10.1097/LBR.0b013e318282d3ca
14. Conforti S, Bonacina E, Ravini M, Torre M. A case of fibrous histiocytoma of the trachea in an infant treated by endobronchial ND:YAG laser. Lung Cancer. 2007;57(1):112-114. doi:10.1016/j.lungcan.2007.01.023

15. Gao H, Ding X, Wei D, et al. Endoscopic management of benign tracheobronchial tumors. $J$ Thorac Dis. 2011;3(4):255-261. doi:10.3978/j.issn.2072-1439.2011.09.02

16. Schumacher B, Charton JP, Nordmann T, Vieth M, Enderle M, Neuhaus H. Endoscopic submucosal dissection of early gastric neoplasia with a water jet-assisted knife: a Western, single-center experience. Gastrointest Endosc. 2012;75(6):1166-1174. doi:10.1016/j.gie.2012.02.027

17. Takeuchi Y, Uedo N, Ishihara R, et al. Efficacy of an endo-knife with a water-jet function (Flushknife) for endoscopic submucosal dissection of superficial colorectal neoplasms. Am J Gastroenterol. 2010;105(2):314-322. doi:10.1038/ajg.2009.547

18. Islas-Garcia JJ, Campos-Salcedo JG, Lopez-Benjume BI, et al. Surgical technique for en bloc transurethral resection of bladder tumour with a Hybrid $\operatorname{Knife}((\mathrm{R}))$. Actas Urol Esp. 2016;40 (4):263-267. doi:10.1016/j.acuro.2015.11.001

19. Li Y, Jiang J, Herth FJF, et al. Primary tracheal mucosa-associated lymphoid tissue lymphoma treated with a water-jet hybrid knife: a case report. Respiration. 2019;97(2):168-172. doi:10.1159/000494064

20. Yin Y, Eberhardt R, Wang XB, et al. Semi-rigid thoracoscopic punch biopsy using a hybrid knife with a high-pressure water jet for the diagnosis of pleural effusions. Respiration. 2016;92(3):192-196. doi: $10.1159 / 000448556$
OncoTargets and Therapy

\section{Publish your work in this journal}

OncoTargets and Therapy is an international, peer-reviewed, open access journal focusing on the pathological basis of all cancers, potential targets for therapy and treatment protocols employed to improve the management of cancer patients. The journal also focuses on the impact of management programs and new therapeutic agents and protocols on patient perspectives such as quality of life, adherence and satisfaction. The manuscript management system is completely online and includes a very quick and fair peer-review system, which is all easy to use. Visit http://www.dovepress.com/ testimonials.php to read real quotes from published authors. 